\title{
Preface to BIT 53:3
}

\author{
Axel Ruhe
}

Published online: 29 August 2013

(C) Springer Science+Business Media Dordrecht 2013

In this penultimate number for 2013, we return to the areas most often covered by BIT, with one linear algebra paper, two contributions to approximation and the remaining eight dealing with differential equations, algorithms for time stepping, finite elements and stochastic equations. I expect few of my readers to go through it all, as I do, but it is interesting to see trends in the BIT subplot of the scientific field.

These are the papers:

John Carroll and Eóin O'Callaghan describe an exponential almost Runge-Kutta method, which is exact for a linear system of ordinary differential equations. It involves following values also of derivative approximations, but the advantage is that it is stable as an implicit method. Its behavior is demonstrated on time stepping for two parabolic problems, one Brusselator and one reaction diffusion advection equation.

Stefan Güttel and Leonid Knizhnerman describe a rational Arnoldi method to compute a matrix function applied to a vector. It is applicable to the class of CauchyStieltjes functions, which contains square roots, logarithms and solutions to certain linear differential equations. It contains an automatic selection of the poles of the rational function and a residual based error estimator. Its behavior is demonstrated on some highly nonnormal test cases, as well as on an applied problem, computing the impedance function of a convection diffusion problem.

Yoshio Komori and Evelyn Buckwar describe how to embed a deterministic RungeKutta method with high order, into a weak order stochastic Runge-Kutta method, and use this to solve a non-commutative stochastic differential equation. Derivative free methods are of special interest, and the method is compared to other available stochastic Runge-Kutta schemes.

\section{A. Ruhe $(\varangle)$}

Numerical Analysis Group, School od Science (SCI), Royal Institute of Technology (KTH), 10044, Stockholm, Sweden

e-mail: ruhe@kth.se 
Gunilla Kreiss and Kenneth Duru study the method of perfectly matched layers to give non reflecting boundary behavior for an anisotropic wave equation. They show that a second order formulation has better stability than the common first order formulation.

Huipo Liu, Wei Gong, Shuanghu Wang, and Ningning Yan solve a Stokes eigenvalue problem by projection into a mixed finite element space. They derive a posteriori error estimates and establish superconvergence.

Fardin Saedpanah solves a hyperbolic integro-differential equation by a continuous space-time finite element method. An a posteriori error analysis is derived.

Bernhard A. Schmitt, Rüdiger Weiner, and Steffen Beck solve an initial value problem with a peer method, where all substeps are of equal order. It is of importance that the output is continuous. The method is tested on two applications of high dimension, to exploit the possibility to take advantage of parallelism.

Ralf Siewer shows that the nodal spline interpolation operator on compact intervals can be regarded as a discretized version of the Martensen operator, designed for twopoint Hermite spline interpolation.

Petr Vabishchevich studies splitting schemes for heat conduction with fast heat transfers, described by a hyperbolic equation. Properties of locally one dimensional splittings are established. It is shown, when an additive difference scheme is unconditionally stable for a fixed staggered grid.

Vesselin Vatchev and Jacqueline Del Castillo study the relation between Hermite and Fejér interpolation in terms of Dirichlet sums approximating a given function.

Eddie Wadbro, Sara Zahedi, Gunilla Kreiss, and Martin Berggren solve an elliptic problem with discontinuities along a surface inside the computational domain with a Nitsche finite element method, that is not sensitive to the position of the interface, relative to the computational grid.

I wish you all welcome to read this new issue,

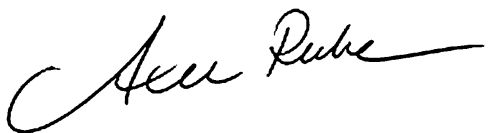

Axel Ruhe 